\title{
Prevalence of abdominal aortic aneurysm and associated lower extremity artery aneurysm in men hospitalized for suspected or known cardiopulmonary disease
}

\author{
Anna Hohneck ${ }^{1,2^{*}}$ (D) Michael Keese ${ }^{3}$, Gerhard Ruemenapf ${ }^{4}$, Klaus Amendt ${ }^{5}$, Hannelore Muertz ${ }^{1}$, Katharina Janda ${ }^{1}$, \\ Ibrahim Akin ${ }^{1,2}$, Martin Borggrefe ${ }^{1,2}$ and Martin Sigl ${ }^{1}$
}

\begin{abstract}
Background: AAA is a disease affecting predominantly male patients $\geq 65$ years and its dreaded complications such as rupture led to population-based screening programs as preventive measure. Nonetheless, the supposed prevalence may have been overestimated, so that targeted screening of high risk populations may be more effective.

This study was performed to evaluate the prevalence of abdominal aortic aneurysm (AAA) of an inpatient high-risk cohort and to estimate the co-prevalence of lower extremity arterial aneurysms.

Methods: Participants: 566 male inpatients, $\geq 65$ years of age, hospitalized for suspected or known cardiopulmonary disease.

Primary and secondary outcome measures: Maximal infrarenal aortic diameters using abdominal ultrasound (leading edge to leading edge method). Upon detection of an AAA (diameter $\geq 30 \mathrm{~mm}$ ), the lower extremity arteries were examined with regard to associated aneurysms.
\end{abstract}

Results: In 40 of 566 patients (7.1\%) AAAs were detectable. Fourteen patients (2.5\%) had a first diagnosis of AAA, none of which was large (>55 mm), the remaining 26 patients were either already diagnosed (14 patients, 2.5\%) or previously repaired (12 patients, 2.1\%).

The three most common main diagnoses at discharge were acute coronary syndrome (43.3\%), congestive heart failure (32.2\%), and chronic obstructive pulmonary disease (12\%). The cohort showed a distinct cardiovascular risk profile comprising arterial hypertension (82.9\%), diabetes mellitus (44.4\%), and a history of smoking (57.6\%).

In multivariate analysis, three-vessel coronary artery disease (Odds ratio (OR): 4.5, 95\% confidence interval (Cl): 2.3-8.9, $p<0.0001)$ and history of smoking (OR: 3.7, Cl: 1.6-8.6, $p<0.01$ ) were positively associated with AAA, while diabetes mellitus (OR: $0.5, \mathrm{Cl}: 0.2-0.9, p=0.0295)$ showed a negative association with AAA.

Among the subjects with AAA, we found two large iliac and two large popliteal aneurysms.

(Continued on next page)

\footnotetext{
* Correspondence: annalena.hohneck@umm.de

${ }^{1}$ First Department of Medicine, University Medical Centre Mannheim, Medical

Faculty Mannheim, University of Heidelberg, Mannheim, Germany

${ }^{2}$ DZHK (German Centre for Cardiovascular Research), partner site Mannheim,

Mannheim, Germany

Full list of author information is available at the end of the article
}

(c) The Author(s). 2019 Open Access This article is distributed under the terms of the Creative Commons Attribution 4.0 International License (http://creativecommons.org/licenses/by/4.0/), which permits unrestricted use, distribution, and reproduction in any medium, provided you give appropriate credit to the original author(s) and the source, provide a link to the Creative Commons license, and indicate if changes were made. The Creative Commons Public Domain Dedication waiver (http://creativecommons.org/publicdomain/zero/1.0/) applies to the data made available in this article, unless otherwise stated. 
(Continued from previous page)

Conclusion: Ultrasound screening in male inpatients, hospitalized for suspected or known cardiopulmonary disease, revealed a high AAA prevalence in comparison to the present epidemiological screening programs. There was a moderate proportion of newly-screen detected AAA and additional screening of the lower extremity arteries yielded some associated aneurysms with indication for possible intervention.

Keywords: Abdominal aortic aneurysm, Screening, High-risk cohort, Lower extremity artery aneurysm

\section{Background}

Implementation of national screening programs of aortic abdominal aneurysms (AAA) in European countries and in the United States of America (USA) were mainly based on data from four large randomized trials initiated in the 1980s and 1990s, reporting an AAA prevalence of $3.9-7.7 \%$ in the male population $>65$ years of age $[18,22,27,28]$. Based on these data, the USA, the United Kingdom (UK) and Sweden implemented national screening programs, with Germany establishing theirs in 2018 [8]. According to the national guidelines in Germany, every man older than 65 years is entitled to a free ultrasound examination of the abdominal aorta. Apart from the above-mentioned countries, there are ongoing trials, such as in New Zealand and Italy $[12,16,21,30]$, supporting screening atrisk populations and also women, in the absence of a formal screening program. Present guidelines also support focused screening of subjects with a distinct risk profile $[6,11]$, as AAA is associated with cardiovascular risk factors, cardiovascular and pulmonary diseases (e.g. COPD), male gender, smoking and age [2, 33, 34].

Screening for AAA has been proven as an effective preventive health measure, which is cost-effective $[35,36]$, though nowadays faced to a declining prevalence of $1-2 \%$ and a decreased incidence of ruptured AAAs in Western countries $[1,5,7,25,31]$.

Against this background, the present study evaluated the prevalence of abdominal aortic aneurysms in a male high-risk cohort ( $\geq 65$ years of age), hospitalized for suspected or known cardiopulmonary disease. Furthermore, we used vascular ultrasound expertise to determine the co-prevalence of associated lower extremity arterial aneurysms.

\section{Methods}

\section{Study design}

By resolution of the GBA (Federal Joint Committee of Insurance Companies and Physicians) of March 2017 and renumeration by statuatory health insurances [26], a nationwide screening program for AAA was established in Germany, starting on January 1, 2018. This study was conducted as direct comparison to the current screening program, to investigate potential benefits of screening a high-risk cohort in an in-hospital setting.

\section{Setting}

This observational study was performed in the First Department of Medicine, University Medical Centre Mannheim, Germany, which is an universitary hospital located in an urban area with a population of over 300.000 inhabitants. Study enrolment started on January 1, 2018 over one year until January 2019. Patients were not followed up.

The study complies with the Declaration of Helsinki and was approved by the local ethical committee, Medical Ethic Commission II, Faculty of Medicine Mannheim, University of Heidelberg, Germany. All patients gave verbal consent to the ultrasound screening and data collection. Data were analyzed anonymously. As the ultrasound examination is part of the clinical routine in cardiovascular medicine without known harmful effects, verbal consent was considered sufficient and approved by the local ethics committee. Data protection was in accordance with the EU Data Protection Directive.

\section{Participants}

Five hundred sixty-six male inpatients ( $\geq 65$ years of age), hospitalized for suspected or known cardiopulmonary disease were enrolled. Patients with advanced oncological concomitant diseases and limited survival rates were excluded. In case of uncertain mental condition or suspicion of low survival rates geriatric assessment was performed.

\section{Variables \\ Data collection}

Data were collected in pseudonymized form. Comorbidities were recorded based on medical history, such as hypertension, hyperlipidaemia, diabetes mellitus and history of smoking. The diagnosis of coronary artery disease (CAD) was proven angiographically. Chronic kidney disease (CKD) was defined as glomerular filtration rate $(\mathrm{GFR})<60 \mathrm{ml} / \mathrm{min}$. The main symptoms leading to hospital admission and the definitive diagnosis of the hospital stay were recorded from the discharge papers, as well as concomitant medication at discharge.

\section{Laboratory parameters}

Blood samples were collected under fasting conditions to determine total cholesterol and LDL-cholesterol. 


\section{Imaging and measurements}

Abdominal aortic ultrasound scans were performed by two experienced angiologists (HM and MS) under fasting conditions. Using a high-end ultrasound scanner (Mindray, Resona 7) with a convex $3.5 \mathrm{MHz}$ transducer, the infrarenal aorta was visualized in its entire length in transverse and longitudinal views. The maximum diameter was measured using the leading edge to leading edge (LELE) method in the plane perpendicular to the arterial axis [15]. AAA was defined by a diameter of $\geq 30$ $\mathrm{mm}$. During the examination vital parameters (heart rate, blood pressure) were recorded. Screening for suprarenal aortic aneurysms was not performed. Upon detection of an AAA (LELE $\geq 30 \mathrm{~mm}$ ), the lower extremity arteries were examined for the presence of iliac, femoral, or popliteal aneurysms. All patients which were newly diagnosed with an AAA were treated according to the present ESC guidelines on the diagnosis and treatment of aortic diseases [11].

\section{Reproducibility}

Prior to patient enrollment a cohort of 40 volunteers was examined by both angiologists (HM and MS) for validation. Inter-observer agreement was determined using Cohen's kappa statistics.

\section{Study size}

A sample size calculation was performed. Assuming an all-over prevalence of ca. $1 \%$ in the population with assuming double the proportion in a high-risk cohort, the estimated sample size would be 474. All patients meeting the inclusion criteria were systematically screened, leading to a number of 614 study participants, whereof 34 were excluded after geriatric assessment, due to uncertain mental condition or suspicion of low survival rates.

\section{Statistical analysis}

Data are presented as mean \pm standard deviation, median (interquartile range), or frequency (percentage). Continuous variables were compared using a two-tailed Student's $t$-test for parametric and Mann-Whitney $U$ test for non-parametric variables. Categorical variables were compared with the $\chi^{2}$ test. Inter-observer agreement for abdominal aortic ultrasound was determined using Cohen's kappa statistics, providing a coefficient ranging from 0 to 1 and its $95 \mathrm{CI}$, with a high value close to 1 indicating high similarity. Multivariate analysis was performed with logistic regression analysis using block entry of the following variables: history of smoking, diabetes mellitus and coronary $3-\mathrm{VD}$, provided to have a $p<0.01$ in univariate analysis.

For all statistical analyses, $p<0.05$ was considered statistically significant. All statistical analyses were performed using Statistical 1 Package for Social Sciences (SPSS version 23, IBM, Munich, Germany).

\section{Results \\ Patients' characteristics}

Six hundred fourteen patients were eligible for inclusion. Thirty-four patients were excluded due to estimated low survival rates $(<2$ years according to geriatric assessment). Fourteen patients were not examined, either because their mental or physical states were considered inadequate, or because they refused the examination after they had been verbally informed regarding possible benefits (early detection) and detriments (anxiety, indication for intervention, risk of intervention). A total of 566 male patients ( $\geq 65$ years of age) was examined. Their median age was 78.0 [72.0-82.0] years. The patients' characteristics are shown in Table 1.

The three most frequent main symptoms leading to hospital admission were angina pectoris, dyspnea, and palpitations/syncope. The three main diagnoses at the time of hospital discharge were acute coronary syndrome (43.3\%), congestive heart failure (32.2\%), or chronic pulmonary disease with or without pneumonia (12.0\%). Comorbidities of the overall cohort included angiographically verified coronary artery disease (CAD, $69.4 \%)$, arterial hypertension (82.9\%), diabetes mellitus (44.4\%), and $57.6 \%$ had a history of smoking.

\section{Findings of the infrarenal aorta}

Visualization of the abdominal aorta was possible in all patients, although a 'second-look'.

ultrasound was required in 3 patients during the hospital stay to obtain adequate measurements. AAAs were detected in 40 out of 566 patients, yielding an overall prevalence of $7.1 \%$. Inter-observer agreement was determined using Cohen's kappa statistics, with a value of 0.98 [0.93 to 1.0$]$, indicating nearly perfect agreement.

The frequencies of previously undetected, already diagnosed, and previously (endovascular or opensurgically) repaired AAAs, as well as the distribution of their sizes (small, medium, or large) are presented in Table 2.

\section{Associations with AAA}

Predictors associated with the presence of AAA in the patients' cohort can be found in Table 3. Univariate analysis revealed an increased prevalence of smoking history $(p<0.001)$ and coronary three-vessel-disease (3-VD) $(p<0.0001)$ and a decreased prevalence of diabetes mellitus $(p<0.01)$. Neither hyperlipidemia $(p=0.110)$, nor arterial hypertension $(p=0.099)$ or chronic kidney disease $(p=0.138)$ were associated with AAA.

Based on the results of the univariate analysis, the following variables were included in the multivariate 
Table 1 Patients' characteristics according to absence or presence of AAA

\begin{tabular}{|c|c|c|c|c|}
\hline & All patients $(n=566)$ & No AAA $(n=526,92.9 \%)$ & $\mathrm{AAA} \geq 30 \mathrm{~mm}(n=40,7.1 \%)$ & $p$-value \\
\hline Age (median, IQR) & $78.0[72.0-82.0]$ & $77.0[72.0-82.0]$ & 78.5 [74.8-83.0] & 0.41 \\
\hline Heart rate (bpm) & $74.9 \pm 14.6$ & $75.0 \pm 14.8$ & $73.4 \pm 12.0$ & 0.51 \\
\hline RR sys (mmHg) & $140.3 \pm 19.3$ & $140.5 \pm 19.2$ & $138.0 \pm 20.7$ & 0.43 \\
\hline RR dia (mmHg) & $80.4 \pm 11.1$ & $80.5 \pm 11.2$ & $79.1 \pm 10.8$ & 0.44 \\
\hline Total cholesterol (mg/dl) & $132.1 \pm 43.9$ & $131.9 \pm 43.8$ & $137.2 \pm 49.7$ & 0.72 \\
\hline LDL (mg/dl) & $91.7 \pm 32.7$ & $91.8 \pm 32.7$ & $88.0 \pm 32.9$ & 0.73 \\
\hline \multicolumn{5}{|l|}{ Comorbidities, n (\%) } \\
\hline Hyperlipidemia & $370(65.4)$ & $339(64.4)$ & $31(77.5)$ & 0.11 \\
\hline Hypertension & $470(83.0)$ & $433(82.3)$ & $37(92.5)$ & 0.10 \\
\hline History of smoking & $327(57.8)$ & $294(55.9)$ & $33(82.5)$ & 0.01 \\
\hline Diabetes mellitus & $251(44.3)$ & $240(45.6)$ & $11(27.5)$ & 0.03 \\
\hline CAD & $393(69.4)$ & $360(68.4)$ & $33(82.5)$ & 0.01 \\
\hline Coronary 3-vessel-disease & $194(34.3)$ & $168(31.9)$ & $26(65.0)$ & $<0.0001$ \\
\hline Chronic kidney disease (GFR $<60 \mathrm{ml} / \mathrm{min})$ & $340(60.1)$ & $311(59.1)$ & $29(72.5)$ & 0.14 \\
\hline \multicolumn{5}{|l|}{ Main diagnosis, $n$ (\%) } \\
\hline ACS & $246(43.5)$ & $224(42.6)$ & $22(55.0)$ & 0.13 \\
\hline Heart failure & $182(32.2)$ & $174(33.1)$ & $8(20.0)$ & 0.09 \\
\hline Exacerbation of COPD/ Pneumonia & $68(12.0)$ & $63(12.0)$ & $5(12.5)$ & 0.92 \\
\hline Others & $31(5.5)$ & $28(5.3)$ & $3(7.5)$ & 0.56 \\
\hline \multicolumn{5}{|c|}{ Main symptoms, leading to hospital admission, $n$ (\%) } \\
\hline Angina pectoris & $166(29.3)$ & $155(29.5)$ & $11(27.5)$ & 0.79 \\
\hline Dyspnea & $208(36.7)$ & $189(35.9)$ & $19(47.5)$ & 0.14 \\
\hline Palpitations/ Syncope & $104(18.4)$ & 99 (18.8) & $5(12.5)$ & 0.32 \\
\hline Others & $35(6.2)$ & $34(6.5)$ & $1(2.5)$ & 0.32 \\
\hline \multicolumn{5}{|l|}{ Concomitant medication, $n$ (\%) } \\
\hline Betablocker & $331(58.5)$ & $308(58.6)$ & $23(57.5)$ & 0.90 \\
\hline Calcium channel blocker & $100(17.7)$ & $96(18.3)$ & $4(10.0)$ & 0.19 \\
\hline ACE inhibitor/ ARB & $444(78.4)$ & $411(78.1)$ & $33(82.5)$ & 0.52 \\
\hline Statin & $375(66.3)$ & $339(64.5)$ & $36(90.0)$ & 0.001 \\
\hline Antiplatelet therapy & $312(55.1)$ & $284(54.0)$ & $28(70.0)$ & $<0.05$ \\
\hline VKA/ DOAC & $190(33.6)$ & 177 (33.7) & $13(32.5)$ & 0.88 \\
\hline
\end{tabular}

CAD coronary artery disease. GFR glomerular filtration rate. $A C S$ acute coronary syndrome. COPD chronic obstructive pulmonary disease. IQR interquartile range

Table 2 Distribution of newly screen-detected, previously diagnosed and previously repaired infrarenal AAA according to the AAA size

\begin{tabular}{llll}
\hline & $\begin{array}{l}\text { Newly screen-detected AAA } \\
(n=14,2.5 \%)\end{array}$ & $\begin{array}{l}\text { Previously diagnosed AAA } \\
(n=14,2.5 \%)\end{array}$ & $\begin{array}{c}\text { Previously repaired AAA } \\
(n=12,2.1 \%)\end{array}$ \\
\hline $\begin{array}{llll}\text { Max. Aortic diameter }(\mathrm{mm})^{a} \\
30-44\end{array}$ & 12 & 10 & 0 \\
$45-54$ & 2 & 4 & $2^{\mathrm{b}}$ \\
$\geq 55$ & 0 & 0 & $10^{\mathrm{b}}$ \\
\hline
\end{tabular}

AAA abdominal aortic aneurysm

${ }^{\text {a }}$ The diameter was measured using the leading edge-to-leading edge (LELE) method

${ }^{b}$ Documented values before the aortic repair were specified 
Table 3 Predictors associated with the presence of AAA in the patients' cohort

\begin{tabular}{lllll}
\hline Predictor & Univariate & $p$-value & Multivariate & OR $(95 \% \mathrm{Cl})$ \\
\hline History of smoking & OR $(95 \% \mathrm{Cl})$ & $3.7(1.6$ to 8.6$)$ & $<0.01$ \\
Diabetes mellitus & $-0.13(-0.11$ to -0.03$)$ & $<0.001$ & 0.2 to 0.9$)$ & 0.03 \\
Coronary 3-VD & $0.12(0.02$ to 0.10$)$ & $<0.01$ & $4.5(2.3$ to 8.9$)$ & $<0.0001$ \\
\hline
\end{tabular}

3-VD three vessel disease. $\mathrm{Cl}$ confidence interval

analysis, which revealed as independent predictors: coronary 3-VD (OR: 4.5, CI: 2.3-8.9, $p<0.0001$ ) and a history of smoking (OR: 3.7, CI: $1.6-8.6, p<0.01$ ) were positively associated with AAA, while diabetes mellitus (OR: 0.5, CI: $0.2-0.9, p=0.0295$ ) showed a negative association with the presence of AAA.

\section{Associated aneurysms}

Among 40 patients with AAA, we found four patients with previously unknown large aneurysms of the lower extremity arteries: two with aneurysms of the common iliac artery $>30 \mathrm{~mm}$, and two with asymptomatic popliteal aneurysms $>20 \mathrm{~mm}$ and poor crural vessel runoff, suggestive of a previous embolism.

\section{Discussion}

Current national population-based screening programs for AAA of all men at or over 65 years have been challenged as the effect of the screening program might be smaller than initially calculated. Therefore we tried to ascertain if focused screening in a high-risk cohort may be more effective.

The main findings of the present study are:

I. The overall prevalence of AAA (> $30 \mathrm{~mm})$ in 566 patients hospitalized for known or suspected cardiopulmonary disease was considerably high (40 patients, $7.1 \%$ ), which can be subdivided into

II. moderate new diagnoses (14 patients, 2.5\%) of AAA, none of which was large (>55 mm), already diagnosed (14 patients, $2.5 \%$ ) or previously repaired AAA (12 patients, 2.1\%).

With Germany establishing the national screening program in 2018, this study was designed as direct comparison, investigating especially the prevalence of AAA in an hospitalized high-risk cohort compared with the general population. A significantly decreasing prevalence of $1-2 \%$ in Western countries in 65-yearold men has been described [7, 32], compared to $3.5 \%$ in the Viborg trial at that age [14]. In our high-risk cohort, we found an overall prevalence of $7.1 \%$, which is comparable with studies in France and Belgium in a similiar setting $[9,19]$.
Following the current German national program for AAA screening, we screened only male inpatients aged $\geq 65$ years. Gianfagna et al. evaluated the AAA prevalence in the general population and subgroups at high cardiovascular risk, including also women and younger subjects [12], which detected an overall prevalence of $1.9 \%$ and peaks of more than $4 \%$ in patients with previous myocardial infarction and ever smokers. In this crosssectional study, patients were randomly selected for ultrasound examination. It would have been interesting to examine also younger men and women in our study, within the in-hospital setting. Even though screening a high-risk population will detect a higher AAA prevalence, it might also detect patients with a lower overall survival and limited life expectancy, reflected by the median age of 78.0 years in our population, and thus questionable benefit. Therefore, targeted screening, also in younger subgroups at higher risk merit evaluation.

AAA and atherothrombotic diseases often coincide, presumably due to related cardiovascular risk factors [29]. In accordance to recent studies we found a strong association between AAA and CAD [10, 20]. In our study, the subgroup of men with angiography-verified CAD revealed an AAA prevalence of 8.4 and $13.4 \%$ among those with three-vessel CAD. Smoking was also found as independent predictor, which is known as the most important modifiable risk factor [16]. Furthermore, we could confirm a "protective effect" of Diabetes mellitus $[3,13]$, which might be explained on one hand by the intensive treatment of patients with diabetes mellitus and a distinct risk profile [24] and on the other may glycated cross-links in the aorta play a protective role [3]. These observations were also made in the REACH Registry [4], which showed a strong association of AAA with a history of smoking and an inverse relation with diabetes. Similiar to the RoCAV population based study [12] the AAA prevalence was evaluated in out-patients with a cardiovascular risk profile. These studies both showed lower prevalences for AAA (2.5\% in the REACH Registry [4] and $1.9 \%$ in the italian population [12]) than in our cohort, which can be explained by the inclusion criteria and the setting. First, the mean age of these study populations was less than 70 years compared with our population with a median age of 78.0 years, as younger patients $(<65$ years) were included. The mentioned 
studies report the prevalence of AAA in out-patients. Screening in-hospital patients could target at-risk individuals who do not participate in national out-patient screening programs. Moreover, women were also included in these two studies. As we conducted our study as direct comparison to the national screening program, we adopted the same criteria, with excluding women. It would have been interesting to screen also hospitalized women, as females have worse prognosis than men and are older at the time of the first diagnosis [17, 23].

The moderate prevalence of $2.5 \%$ of previously unknown AAA must be seen in the context of the following: the majority of the patients were in frequent contact with the health system before the index hospital admission. AAAs may be detected as incidental finding within routine diagnostic abdominal imaging. Furthermore, the implementation of population-based screening programs is supposed to decrease the in-hospital detection rates of previously unknown AAAs. In our cohort, 14 patients had a first diagnosis of AAA, which is approximately double the proportion of AAAs in current populationbased screening programs of Western countries. We did not find any large AAAs $(>55 \mathrm{~mm})$ among the newlyscreen detected.

Furthermore, we performed additional screening for associated aneurysms of the lower extremity arteries in all patients with an AAA, independent of the time of the diagnosis. Among 40 patients with AAAs, 4 patients had previously unknown large aneurysms of their lower extremity arteries, yielding a prevalence of $10 \%$, which is in accordance to the literature (37), with expecting even higher prevalence in the entire study cohort, though systematic screening has not been performed.

Our study supports the usefulness of screening subgroups at high cardiovascular risk. However, as patients were not followed, a cost-effectiveness evaluation was not performed, which limited the interpretation of the results.

\section{Strengths and limitations of this study}

- Ultrasound screening for AAA was performed in a high-risk inpatient cohort (men $>65$ years of age) using the selection criteria of current populationbased national screening programs.

- In those patients admitted to our institution, we identified AAA that were previously unknown, diagnosed, or repaired.

- Furthermore, all patients with any AAAs were systematically screened for AAA-associated lower extremity arterial aneurysms.

- The lack of long-term follow-ups and costeffectiveness evaluations limited the interpretation of the results and does not contribute to the benefitversus-harm discussion in AAA screening.

\section{Conclusion}

Ultrasound screening in male inpatients, hospitalized for suspected or known cardiopulmonary disease, revealed a high AAA prevalence AAA in comparison to the present epidemiological screening programs. Furthermore, additional systematically screening of the lower extremity arteries yielded a relevant number of associated aneurysms with indication for possible intervention.

Performing AAA screening in a cardiopulmonary inpatient cohort combines high prevalence of cardiovascular risk factors and vascular ultrasound expertise. Screening this high-risk cohort for AAA and associated aneurysm would be feasible.

\section{Abbreviations \\ 3-VD: Three-vessel-disease; AAA: Abdominal aortic aneurysm; ACS: Acute coronary syndrome; CAD: Coronary artery disease; Cl: Confidence interval; COPD: Chronic obstructive pulmonary disease; GFR: Glomerular filtration rate; IQR: Interquartile range; LELE: Leading edge to leading edge; PAA: Popliteal artery aneurysm}

\section{Acknowledgements}

The authors would like to thank Dr. Christel Krahl, Marianne Hoffmann and Andrea Wielsch for their time and support regarding the care of patients. We also would like to thank our patient advisers for their contribution to the study design.

\section{Authors' contributions}

$\mathrm{AH}$ collected and analyzed all data and made a decisive contribution to completion of the manuscript. MK, GR, KA, IA and MB provided critical revisions for intellectual content, and HM performed a high percentage of the ultrasound examinations. $\mathrm{KJ}$ helped collect and analzse the data and wrote parts of the manuscript. MS conceived the study, performed a majority of the ultrasound examinations, approved the statistical analysis and prepared the initial manuscript draft. All authors approved the final version.

\section{Funding}

We acknowledge financial support by Deutsche Forschungsgemeinschaft (German Research Foundation) within the funding programme Open Access Publishing, by the Baden-Württemberg Ministry of Science, Research and the Arts and by Ruprecht-Karls-Universität Heidelberg. The funding body was not involved in the conception or conducting of the study or in writing the manuscript.

Availability of data and materials

Datasets are available from the corresponding author upon reasonable request.

\section{Ethics approval and consent to participate}

The study complies with the Declaration of Helsinki and was approved by the local ethical committee, Medical Ethic Commission II, Faculty of Medicine Mannheim, University of Heidelberg, Germany. All patients gave verbal consent to the ultrasound screening and data collection. Data were analyzed anonymously. As the ultrasound examination is part of the clinical routine in cardiovascular medicine without known harmful effects, verbal consent was considered sufficient and approved by the local ethics committee. Data protection was in accordance with the EU Data Protection Directive.

Consent for publication

Not applicable

\section{Competing interests}

Ibrahim Akin is a member of the editorial board of BMC Cardiovascular Disorders. 


\section{Author details}

First Department of Medicine, University Medical Centre Mannheim, Medical Faculty Mannheim, University of Heidelberg, Mannheim, Germany. ${ }^{2}$ DZHK (German Centre for Cardiovascular Research), partner site Mannheim, Mannheim, Germany. ${ }^{3}$ Department of Vascular Surgery, University Medical Centre Mannheim, Medical Faculty Mannheim, University of Heidelberg, Mannheim, Germany. ${ }^{4}$ Department of Vascular Surgery, Diakonissen-Stiftungs-Krankenhaus Speyer, Speyer, Germany. ${ }^{5}$ Department of Angiology, Cardiology and Diabetes associated diseases, Diakonissenkrankenhaus Mannheim, Gefäßzentrum Oberrhein, Mannheim, Germany.

Received: 16 August 2019 Accepted: 15 November 2019 Published online: 09 December 2019

\section{References}

1. Abdulameer H, Al Taii H, Al-Kindi SG, Milner R. Epidemiology of fatal ruptured aortic aneurysms in the United States (1999-2016). J Vasc Surg. 2019;69(2):378-384. e372.

2. Altobelli E, Rapacchietta L, Profeta V, Fagnano R. Risk factors for abdominal aortic aneurysm in population-based studies: a systematic review and metaanalysis. Int J Environ Res Public Health. 2018;15(12):2805.

3. Avdic T, Franzén S, Zarrouk M, Acosta S, Nilsson P, Gottsäter A, et al. Reduced Long-term risk of aortic aneurysm and aortic dissection among individuals with type 2 diabetes mellitus: a Nationwide observational Study. J Am Heart Assoc. 2018;7(3):e007618.

4. Baumgartner I, Hirsch AT, Abola MTB, Cacoub PP, Poldermans D, Steg PG, et al. Cardiovascular risk profile and outcome of patients with abdominal aortic aneurysm in out-patients with atherothrombosis: data from the Reduction of Atherothrombosis for Continued Health (REACH) Registry. J Vasc Surg. 2008;48(4):808-814. e801.

5. Benson RA, Poole R, Murray S, Moxey P, Loftus IM. Screening results from a large United Kingdom abdominal aortic aneurysm screening center in the context of optimizing United Kingdom National Abdominal Aortic Aneurysm Screening Programme protocols. J Vasc Surg. 2016;63(2):301-4.

6. Chaikof EL, Dalman RL, Eskandari MK, Jackson BM, Lee WA, Mansour MA, et al. The Society for Vascular Surgery practice guidelines on the care of patients with an abdominal aortic aneurysm. J Vasc Surg. 2018;67(1):2-77. e72.

7. Choke E, Vijaynagar B, Thompson J, Nasim A, Bown MJ, Sayers RD. Changing epidemiology of abdominal aortic aneurysms in England and Wales: older and more benign? Circ. 2012;125(13):1617-25.

8. Debus E. S3-Leitlinie zum Screening, Diagnostik Therapie und Nachsorge des Bauchaortenaneurysmas. Springer; 2018

9. Durieux R, Van Damme H, Labropoulos N, Yazici A, Legrand V, Albert A, et al. High prevalence of abdominal aortic aneurysm in patients with threevessel coronary artery disease. Eur J Vasc Endovasc Surg. 2014;47(3):273-8.

10. Elkalioubie A, Haulon S, Duhamel A, Rosa M, Rauch A, Staels B, et al. Metaanalysis of abdominal aortic aneurysm in patients with coronary artery disease. Am J Cardiol. 2015;116(9):1451-6.

11. members ATF, Erbel R, Aboyans V, Boileau C, Bossone E, Bartolomeo RD, et al. 2014 ESC guidelines on the diagnosis and treatment of aortic diseases: document covering acute and chronic aortic diseases of the thoracic and abdominal aorta of the adult the task force for the diagnosis and treatment of aortic diseases of the European Society of Cardiology (ESC). Eur Heart J 2014:35(41):2873-2926

12. Gianfagna F, Veronesi G, Tozzi M, Tarallo A, Borchini R, Ferrario MM, et al. Prevalence of abdominal aortic aneurysms in the general population and in subgroups at high cardiovascular risk in Italy. Results of the RoCAV population based study. Eur J Vasc Endovasc Surg. 2018;55(5):633-9.

13. Golledge J, Muller J, Daugherty A, Norman P. Abdominal aortic aneurysm: pathogenesis and implications for management. Arterioscler Thromb Vasc Biol. 2006;26(12):2605-13.

14. Collaborative Aneurysm Screening Study Group (CASS Group), Chichester Aneurysm Screening Group (UK), Viborg Aneurysm Screening Study (Denmark), Western Australia Abdominal Aortic Aneurysm Program (Australia), \& Multicentre Aneurysm Screening Study (MASS)(UK). A comparative study of the prevalence of abdominal aortic aneurysms in the United Kingdom, Denmark, and Australia. J Med Screen. 2001;8(1):46-50.

15. Gürtelschmid M, Björck M, Wanhainen A. Comparison of three ultrasound methods of measuring the diameter of the abdominal aorta. Br J Surg. 2014;101(6):633-6.
16. Jones G, Hill B, Curtis N, Kabir TD, Wong L, Tilyard M, et al. Comparison of three targeted approaches to screening for abdominal aortic aneurysm based on cardiovascular risk. Br J Surg. 2016;103(9):1139-46.

17. Lederle FA, Johnson GR, Wilson SE. Abdominal aortic aneurysm in women. J Vasc Surg. 2001;34(1):122-6.

18. Lindholt JS, Vammen S, Juul S, Henneberg E, Fasting $H$. The validity of ultrasonographic scanning as screening method for abdominal aortic aneurysm. Eur J Vasc Endovasc Surg. 1999;17(6):472-5.

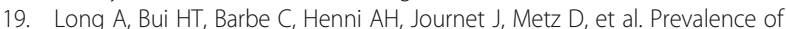
abdominal aortic aneurysm and large infrarenal aorta in patients with acute coronary syndrome and proven coronary stenosis: a prospective monocenter study. Ann Vasc Surg. 2010;24(5):602-8.

20. Madaric J, Vulev I, Bartunek J, Mistrik A, Verhamme K, De Bruyne B, et al. Frequency of abdominal aortic aneurysm in patients $>60$ years of age with coronary artery disease. Am J Cardiol. 2005:96(9):1214-6.

21. Majeed K, Hamer A, White S, Pegg T, Wilkins G, Williams S, et al. Prevalence of abdominal aortic aneurysm in patients referred for transthoracic echocardiography. Intern Med J. 2015;45(1):32-9.

22. Norman P, Castleden W, Hockey R. Prevalence of abdominal aortic aneurysm in Western Australia. Br J Surg. 1991;78(9):1118-21.

23. Norman P, Powell J. Abdominal aortic aneurysm: the prognosis in women is worse than in men. Circ. 2007:115(22):2865-9.

24. Patel K, Zafar MA, Ziganshin BA, Elefteriades JA. Diabetes mellitus: is it protective against aneurysm? A narrative review. Cardiol. 2018;141(2): 107-22.

25. Robert $M$, Juillière $Y$, Gabet $A$, Kownator $S$, Olié $V$. Time trends in hospital admissions and mortality due to abdominal aortic aneurysms in France, 2002-2013. Int J Cardiol. 2017;234:28-32.

26. Schmitz-Rixen T. Ultraschalluntersuchung zur Früherkennung von Bauchaortenaneurysmen. Springer; Gefässchirurgie. 2017;22:4. https://doi. org/10.1007/s00772-016-0235-6.

27. Scott R, Group MASS. The multicentre aneurysm screening Study (MASS) into the effect of abdominal aortic aneurysm screening on mortality in men: a randomised controlled trial. Lancet. 2002;360(9345):1531-9.

28. Scott R, Wilson N, Ashton H, Kay D. Influence of screening on the incidence of ruptured abdominal aortic aneurysm: 5-year results of a randomized controlled study. Br J Surg. 1995:82(8):1066-70

29. Singh K, Bønaa K, Jacobsen B, Bjørk L, Solberg S. Prevalence of and risk factors for abdominal aortic aneurysms in a population-based study: the Tromsø Study. Am J Epidemiol. 2001;154(3):236-44.

30. Stather P, Dattani N, Bown M, Earnshaw J, Lees T. International variations in AAA screening. Eur J Vasc Endovasc Surg. 2013;45(3):231-4.

31. Svensjö S, Björck M, Gürtelschmid M, Djavani Gidlund K, Hellberg A, Wanhainen A. Low prevalence of abdominal aortic aneurysm among 65year-old Swedish men indicates a change in the epidemiology of the disease. Circ. 2011:124(10):1118-23.

32. Sweeting MJ, Oliver-Williams CT, Thompson SG. Lessons learned about prevalence and growth rates of abdominal aortic aneurysms from a 25-year ultrasound population screening programme. 2018.

33. Takagi $\mathrm{H}$, Umemoto T. ALICE (all-literature investigation of cardiovascular evidence group). A meta-analysis of adjusted observational studies and randomized controlled trials of endovascular versus open surgical repair for ruptured abdominal aortic aneurysm. Int Angiol. 2016;35(6):534-45.

34. Takagi $H$, Umemoto T. Associations of coronary and peripheral artery disease with presence, expansion, and rupture of abdominal aortic aneurysm-a grin without a cat! VASA. 2017;46(3):151-8.

35. Wanhainen A, Hultgren R, Linné A, Holst J, Gottsäter A, Langenskiöld M, et al. Outcome of the Swedish nationwide abdominal aortic aneurysm screening program. Circ. 2016:134(16):1141-8.

36. Zarrouk M, Lundqvist A, Holst J, Troëng T, Gottsäter A. Cost-effectiveness of screening for abdominal aortic aneurysm in combination with medical intervention in patients with small aneurysms. Eur J Vasc Endovasc Surg. 2016:51(6):766-73.

\section{Publisher's Note}

Springer Nature remains neutral with regard to jurisdictional claims in published maps and institutional affiliations. 